\title{
Challenges and solutions for MV and LV protection in grids with large amount of distributed generation - a final report from the German research project ProFuDis
}

\author{
Felix Glinka ${ }^{1}$, Tilman Wippenbeck ${ }^{1}$, Thomas Schmidt ${ }^{2}$, \\ Thomas Wiedemann ${ }^{2}$, Christoph Bennauer ${ }^{3}$ \\ 'IFHT, RWTH Aachen University, Aachen, Germany \\ ${ }^{2}$ Innogy SE, Essen, Germany \\ ${ }^{3}$ Schneider Electric GmbH, COE AED, Seligenstadt, Germany \\ $\bowtie$ E-mail: felix.glinka@rwth-aachen.de
}

\begin{abstract}
The ongoing installation of distributed generation (DG) in low- (LV) and medium-voltage (MV) grids might be prospectively limited by restrictions of today's protection systems due to the DG's impact onto the system's fault behaviour. Investigations show that today's DG shares do not yet lead to protection malfunctions. A reasonable future increase of the share of DG and the increasing complexity of the grids will partially pose protection-challenges in the near future though. Therefore, detailed protection planning becomes increasingly important in the future, using enhanced calculation, modelling and evaluation approaches, as developed within the project Protection for Future Distribution Systems (ProFuDiS). While the future protection-challenges in German MV grids can widely be solved using altered parametrisation and application of the present digital protection devices, the usage of classical $\mathrm{NH}$ fuses for LV grid protection will be possible for most, but not all grids any more. While sufficient, but rather complex digital protection approaches are already available, the development of innovative, more cost-efficient solutions for LV protection should be carried on. Additionally more precise regulations for the fault behaviour of DG should be stated to allow effective protection planning and reliable and realistic fault simulation.
\end{abstract}

\section{Introduction}

The continuous increase of distributed generation (DG) in Germany leads to altered current and voltage characteristics in case of grid faults, especially due to the predominant inverter coupling of DG [1]. Within the research project 'Protection for Future Distribution Systems' (ProFuDiS) the impact of DG onto the protection concepts of distribution grids and the applicability of today's approaches is investigated. A group of 10 partners is contributing to this project, formed by universities (HTW Saar, IFHT/RWTH Aachen), a research facility (FGH e.V.), a distribution system operator (innogy) and industrial partners (SMA, NH-HH Recycling, OMICRON, ABB, Schneider electric, Siemens) Fig. 1.

\section{German DG regulations}

The behaviour of DG within the German medium-voltage (MV) and low-voltage (LV) grids is regulated and differs regarding the reaction to grid faults. Today DG in LV grids are required to disconnect within $200 \mathrm{~ms}$ in case of a voltage drop at their point of common coupling (PCC) below $0.8 V_{N}$ residual voltage [2]. [3]:

DG in the German MV grids is required to be technically ready to

- 'remain connected to the grid in case of a fault',

- 'inject a defined reactive current during the fault to support the grid voltage'.

The actual behaviour regarding the reactive current injection is defined by the DSO.
Due to the expected decrease of power produced by large power plants, it is currently being discussed to apply the MV requirements also to the LV area in order to provide an additional reactive current as grid support [4].

\section{Fault behaviour of inverters}

The guidelines leave certain levels of freedom for the actual realisation within the DG. Especially the behaviour of inverter interfaced DG (IIDG) is severely dependent on the manufacturer and type-specific control. Therefore, it is very flexible. One example is the active current infeed during dynamic grid support. It is not defined in the present guidelines and can reach from a maximisation of the total current to a reduction of the active current, i.e. zero (Fig. 2). Experiments in IFHT with conventional IIDG prove the shown behaviour. Additionally the dynamic behaviour of IIDGs differs considerably. The settling times of the active and in particular the reactive currents are measured to up to $150 \mathrm{~ms}$. Furthermore, IIDGs are showing phase jumps while reacting to sudden changes e.g. in the grid voltages. Additionally a high pollution of the infeed currents with harmonics during grid faults can be measured. All of these effects have possible impact onto the functionality of today's protection systems and need to be regarded as uncertainties when calculating short circuit currents [1].

\section{$4 \quad$ IIDG impact onto German LV grids}

Most German LV grids are protected using NH fuses. These fuses ensure a reliable functionality for currents exceeding the conventional fusing current $\left(1.6 I_{\mathrm{NH}}\right)$. The intermediate infeed of 


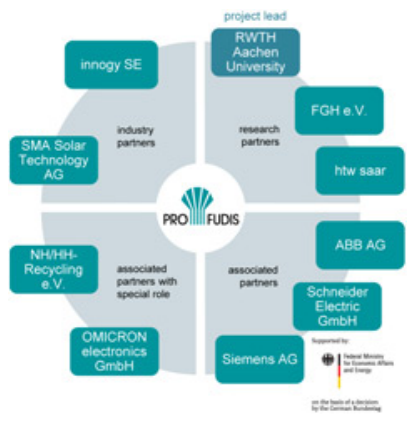

Fig. 1 ProFuDiS project partners

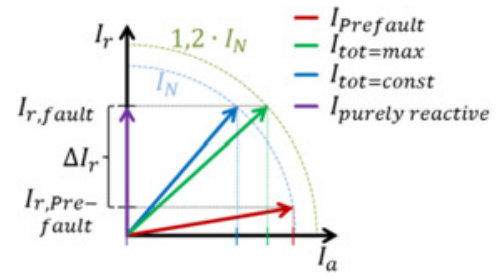

Fig. 2 Variants of active current behaviour during dynamic grid support

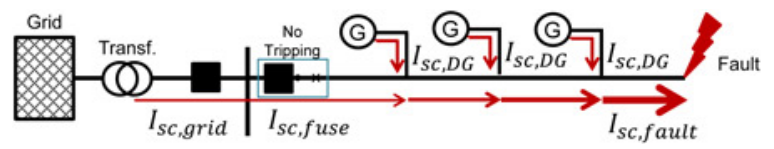

Fig. 3 Blinding in $L V$ grids

DG in LV grids can potentially lower the short circuit currents through the fuse, leading to an increase in tripping time up to a failure to operate (Fig. 3) [5].

Extensive parameter studies as well as laboratory experiments with conventional equipment show a potential thread for blinding in realistic setups [5]. Based on these studies a first evaluation of today's LV grids of a large German DSO is carried out. The results show a share of $0.3 \%$ of potentially affected LV grids. One of them is presented in Fig. 4.

The grid consists of a total line length of about $790 \mathrm{~m}$ NAYY $4 \times 70$ SE cable. The line is divided into two protection zones according to the present DSO's guideline, while zone one is protected using a $200 \mathrm{~A} \mathrm{NH} 2 \mathrm{gG}$ fuse, zone two uses a $125 \mathrm{~A}$ $\mathrm{NH} 2 \mathrm{gG}$ fuse. Within zone one, there are four IIDG installed with an actual total power of $92 \mathrm{~kW}$. Additionally to that case, a variation of different IIDG power-distributions is considered. Short circuit variation calculations are conducted, varying the fault type, the fault impedance as well as the fault position.

All faults in zone two are reliably cleared, also with IIDG. In the following only those results are regarded, for which the IIDGs stay in a normal state according to [2], and therefore do not shut off in the passive grid. Apart from that only those fault cases are regarded, which lead to a fault current of $1.6 \times I_{\mathrm{NH}, 200 \mathrm{~A}}=320 \mathrm{~A}$ without IIDG and are therefore reliably turned off. Fig. 5 shows the

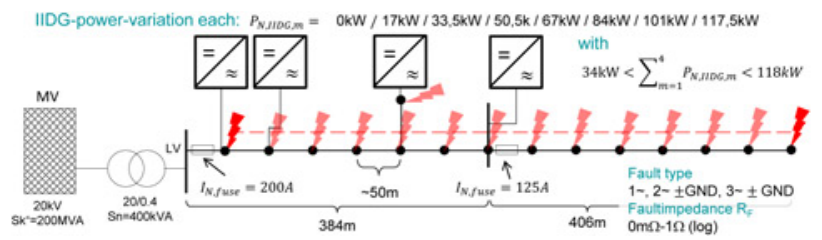

Fig. 4 Real LV grid for blinding investigation

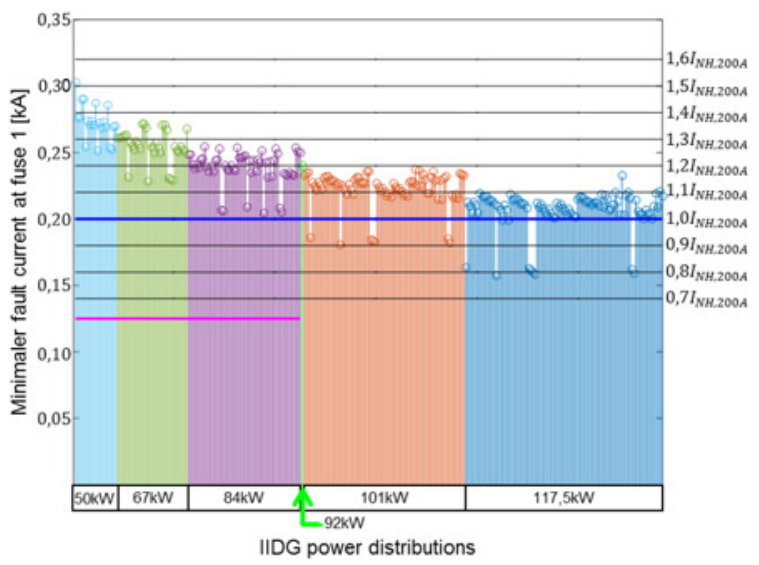

Fig. 5 Minimum fault currents with IIDG

minimum fault currents with IIDGs. It can be seen that the minimum fault current is below $320 \mathrm{~A}$ for all configurations, and therefore would not be discovered by the $200 \mathrm{~A} \mathrm{NH}$ fuse and lead to blinding.

Different solutions can be taken to alter the protection of the line in order to ensure a proper protection with IIDG. One solution can be the usage of an $\mathrm{NH}$ fuse with a smaller nominal current rating.

For the configurations up to $84 \mathrm{~kW}$ a $125 \mathrm{~A}$ fuse with a conventional fusing current of $200 \mathrm{~A}$ would ensure a proper protection. Also the usage of enhanced $\mathrm{NH}$ fuses with a minimum fusing current of $1.4 \times I_{\mathrm{NH}, N}$ can pose a suitable solution.

For a simplified selection of the NH fuses in LV single line topologies the minimum short circuit current without IIDG, according to the present guidelines, subtracted with the total installed IIDG peak current can be used as a worst case estimation:

$$
I_{\mathrm{sc}, \text { min }, \text { fuse }}=I_{\mathrm{sc}, \text { min ,noIIDG }}-I_{\text {peak,IIDG,tot }}
$$

Another possible solution is the usage of a (directional) overcurrent relay. It allows a narrow gap between the nominal and the minimum pickup current. For directional relays even a minimum pickup current below the maximum infeed current can be parameterised as long as the maximum load current is not exceeding the minimum fault current with blinding. As a switching element either LV switchgears can be used, which mostly lead to fairly high investment and construction during retrofit. Another possibility is the usage of triggered fuses [6] as commercially available for DC systems [7]. A prototype of a 100 A trigger fuse was successfully tested in a blinding situation with a fault current of $90 \mathrm{~A}$ for an $\mathrm{AC}$ grid topology in the testing lab of the IFHT. The trigger is realised using a directional overcurrent relay. Total shut off times of $<100 \mathrm{~ms}$ are achieved. Trigger fuses have the advantage of fitting into existing NH-fuse mountings. Furthermore, the triggered fuses provide the conventional fuse characteristic for high current faults. For a wide usage of trigger fuses, further research needs to be done, especially to find cheap and small digital detection devices to trigger the fuse.

For the shown example all faults can be cleared with the help of a directional overcurrent detection with a thresh-old of $0.7 \times I_{\mathrm{NH}, 200 \mathrm{~A}}=140 \mathrm{~A}$ (Fig. 6). Nevertheless, most configurations could already be cleared using a non-directional threshold of $200 \mathrm{~A}$. This leads to the conclusion that a detailed analysis of grids with high IIDG penetration should be done using proper calculation methods in order to select the most suitable and efficient protection.

\section{$5 \quad$ IIDG impact onto German MV grids}

Digital non-directional overcurrent relays are widely used in German MV grids as primary protection. Similar to the impact in the LV area 


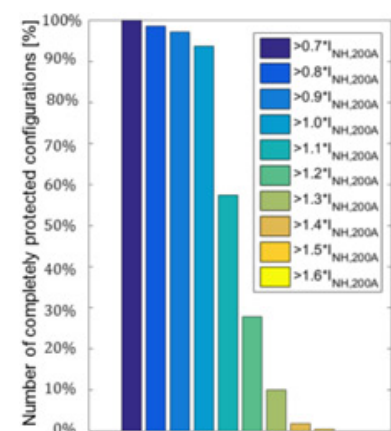

Fig. 6 Number of protected distributions

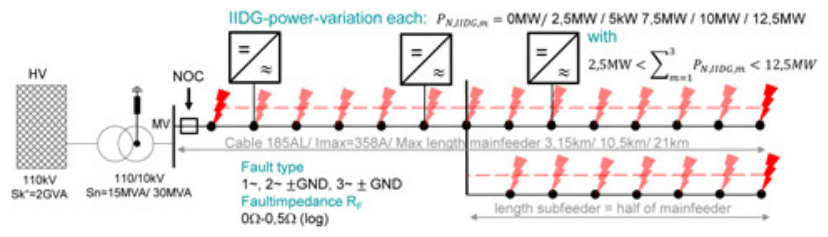

Fig. 7 Parameter study for blinding in a typical German MV grid structure

the infeed of IIDG can affect the functionality of the protection relays due to the changes in short circuit currents. Therefore, short circuit current calculations in combination with parameter studies are carried out. The grid topologies and assets investigated are based on typical characteristics of the MV grids of a large German DSO. One of the investigated setups is shown in Fig. 7.

The IIDGs are parameterised according to [3] and deliver a symmetric short circuit current with an additional reactive component in dependence of the residual voltage at their PCC. At the same time the IIDG are parameterised to maximize their active power infeed up to a total current of $I_{\text {max,IIDG,tot }}=1.2_{\text {Ipeak,IIDG. In }}$ order to be able to consider the current source behaviour of the IIDGs a steady state short circuit current calculation method based on current superposition is used [1].The results show a reduction of the current at the position of the protection relay by up to $45 \%$ in comparison to the corresponding calculation without IIDG infeed. In order to determine the significant influencing factors of the blinding effect, the thousand most critical blinding cases of the parameter studies were evaluated in Fig. 8. Furthermore, it can be seen that the infeed behaviour of the IIDG can lead to situations in which the fault current for three phase faults falls below the corresponding fault current for two-phase faults. Therefore the common assumption of the two-phase fault being the most critical in terms of the minimum fault current is not valid any longer and should not be used any more as a criterion for the protection relay parametrisation in grids with high IIDG share. Similar to the results in the LV grid the impact of the IIDG installed in the beginning of the line can be considered more severe.

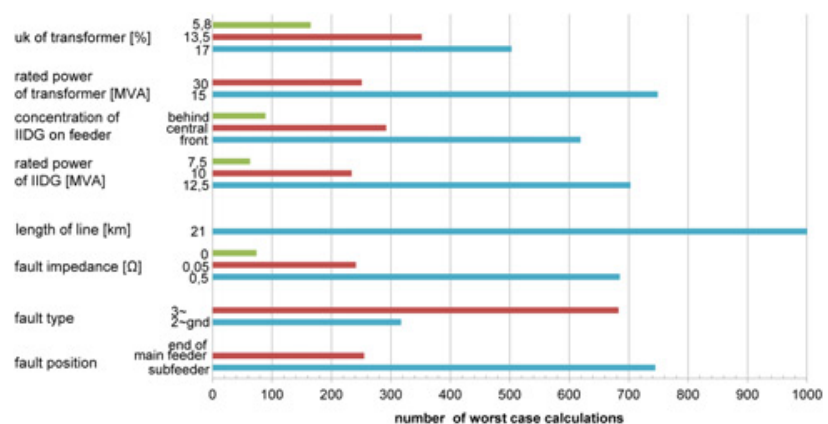

Fig. 8 Parameter study results: Factors influencing blinding
Matching the results of the parameter studies with the actual grids and settings for the overcurrent relays in the grids of the German DSO Innogy SE does not show protection problems today and in the near future. The gap between the maximum nominal currents and the resulting minimum fault currents, even with IIDGs, allows a proper parametrisation in most of the present grids. Still the protection in $10 \mathrm{kV}$ systems should be investigated more closely in grids with combinations of the total line length and the total nominal power of the IIDG exceeding:

$$
\begin{aligned}
& L_{\max } \geq 15 \mathrm{~km} \text { and } S_{\text {IIDG,tot }} \geq 12 . \text { MVA, or } \\
& L_{\max } \geq 20 \mathrm{~km} \text { and } S_{\text {IIDG,tot }} \geq 7 . \text { MVA }
\end{aligned}
$$

Especially for long radial feeders with a large amount of installed IIDGs, the non-directional overcurrent relays are not suitable any more.

\section{Reliability of short-circuit direction determination}

Directional overcurrent protection relays (DOC) may be a solution for long radial feeders in future distribution systems with massive integration of IIDG (Fig. 9).

The blinding effect may cause that the operating current for reverse infeed into the feeding grid (blue) becomes higher than the related minimum short-circuit fault current in forward direction (red). Reliability of protection by a DOC is then dependent onto the reliability of the short circuit direction determination (SCDD). For down-stream faults, a wrong directional decision may lead to a failure to operate. The intended usage of DOC as a solution for future overcurrent protection challenges requires answering the following questions beforehand:

- How reliably will SCDD of commercially available DOC operatein the new application scenario?

- How can feeders at risk be identified in practice?

- Which solutions can be found?

In the following investigation a realistic rural $20 \mathrm{kV}$ distribution grid is selected (Fig. 10). Due to its feeder line length and impedance, the short-circuit current of two-phase faults at the line end is relatively low. Multiple IIDGs are installed near to the

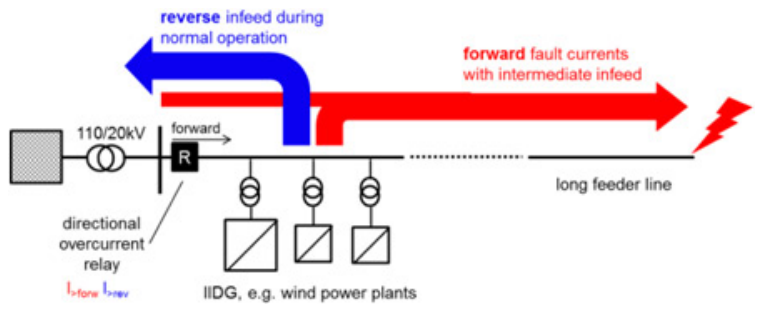

Fig. 9 New application scenario for DOC protection as a solution for long radial feeders with high levels of IIDG

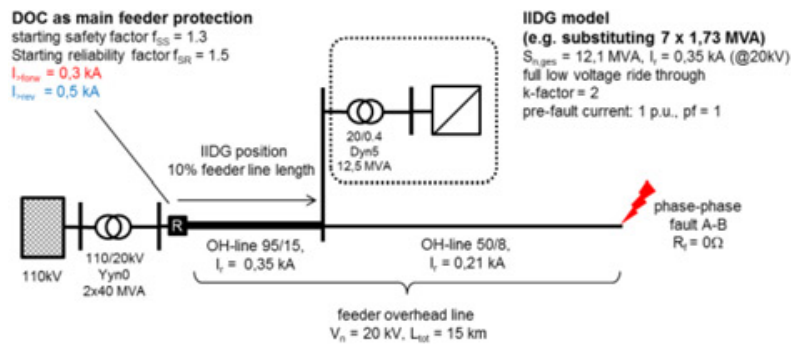

Fig. 10 Study case data. IIDG according to [8] 


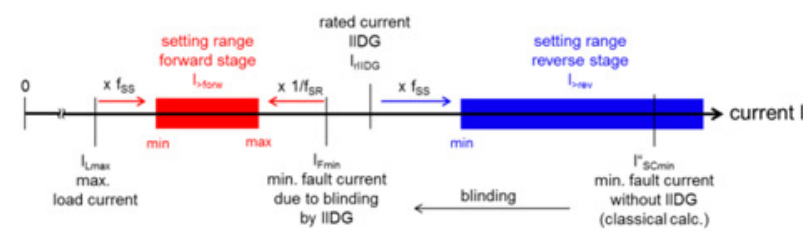

Fig. 11 Derivation of permissible setting ranges for DOC stages in forward and reverse direction

busbar with a total infeed of about $S_{\mathrm{n}} ; 12$ MVA. The study case is modelled in a steady state tool previously developed for Matlab ${ }^{\circledR}$ [1]. The IIDG provide full voltage ride through and behave according to [8]. The simulation parameters used are given in Fig. 10.

A DOC is used as relay $\mathrm{R}$ with a forward (in $\operatorname{red} I>_{\text {forw }}$ ) and reverse (in blue $I>_{\text {rev }}$ ) stage setting.

The related minimum setting thresholds in forward and reverse direction (Fig. 11) are determined by the minimum load current $\left(I_{\mathrm{Lmax}}\right)$ and the summed nominal infeed cur-rent of the IIDG $\left(I_{\text {rIIDG }}\right)$ multiplied by the starting security factor $f_{\text {SS }}$ (typical value for $f_{\mathrm{SS}}=1.3$ ).

Without IIDG the minimum short-circuit current $\Gamma_{\mathrm{k}, \min }$ in forward direction may be calculated according to established standards [9]. Due to the intermediate infeed the minimum fault current at the measuring point of $\mathrm{R}$ may get reduced to $I_{\mathrm{Fmin}}=I_{\text {SCmin }} I_{\mathrm{rIIDG}}$ (worst-case estimation). The maximum limit of the setting range is decreased further by the starting reliability factor $f_{\mathrm{SR}}$ $\left(I_{>\text {forw } \max }=I_{\text {Fmin }} / f_{\text {SR }}\right.$, typically $\left.f_{\mathrm{SR}}=1.5\right)$.

Fig. 12 shows the calculated currents and permissible setting ranges of the DOC in the grid given by Fig. 10. The blinding effect necessitates the usage of a DOC as the forward (red bars) and backward (blue bar) permissible setting ranges do not overlap. The current in phase $\mathrm{C}$ exceeds the chosen $I_{>\text {forw }}$ in Fig. 12 (red line), although only phases A and B are affected by the fault. This current flow in the healthy phase $\mathrm{C}$ distinguished the new application scenario from fault cases without IIDG.

To evaluate SCDD reactions, models of three commercial DOCs with different SCDD variants according to Table 1 are implemented in Matlab ${ }^{\circledR}$. A study is performed varying the study case parameters given in Table 2 .

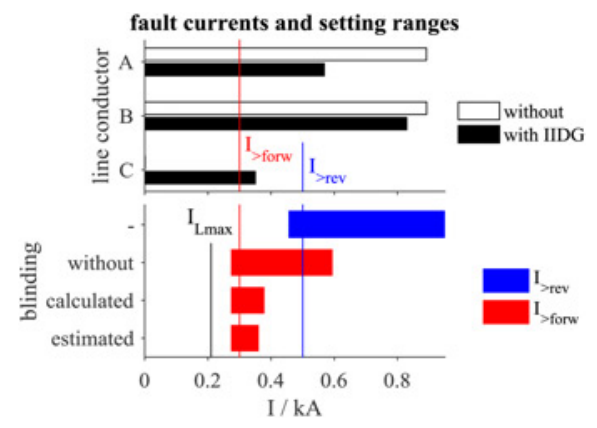

Fig. 12 Fault currents and permissible setting ranges of the DOC considering the blinding effect

Table 1 Direction evaluation criteria of DOC relays

\begin{tabular}{lcc}
\hline SCDD & Criteria & Polarisation quantity \\
\hline 1 & current to voltage angles & fault case-dependant \\
$2 \mathrm{a}$ & & self-polarisation \\
$2 \mathrm{~b}$ & & external polarisation \\
$2 \mathrm{c}$ & active power sign & positive sequence polarisation \\
$3 \mathrm{a}$ & & total power \\
$3 \mathrm{~b}$ & & positive sequence power \\
\hline
\end{tabular}

Table 2 Most relevant subset of varied simulation parameters of the example study case

\begin{tabular}{lc}
\hline Parameter & Values \\
\hline feeder line length & $10 / 15 / 20 \mathrm{~km}$ \\
position of IIDG (Fig. 1) & $5 / 10 / 25 \%$ \\
total rating of IIDG and coupling & within reasonable application \\
transformer (total rated current, & boundaries DOC: $I_{\text {IIIDG }}=(0.34-0.495)$ \\
respectively) & I" $\mathrm{SC}$, min \\
pre-fault current IIDG & $0 / 50 / 100 \%$ \\
$k$-factor of IIDG dynamic grid & $0 / 1 / 2$ \\
stabilisation [8] & \\
\hline
\end{tabular}

For three-phase (3p) faults with intermediate IIDG infeed and any fault case without IIDG all SCDD provide a reliable direction determination. In case of line-line faults (2p) Fig. 13 shows that the some SCDD may determine a false direction or may suffer to determine one.

Those cases are investigated further. SCDD 1 partially interprets the fault as $3 p$ instead of $2 p$ due to the current in phase C. The SCDD then selects wrong measuring values and characteristic angles for the direction determination. The SCDD $2 \mathrm{a}-\mathrm{c}$ in some cases does not provide a directional decision at all due to inner discrepancies caused by the phase $\mathrm{C}$ current. The actual SCDD behaviour then depends on predefined polarisation. Critical cases for SCDD 1-2 are related to settings of $I_{>\text {forw }}<I_{\text {rIIDG }}$. SCDD $3 \mathrm{a}-\mathrm{b}$ show failure rates of $<2 \%$.

In applications that need a setting of $I_{>\text {forw }}<I_{\text {rIIDG }}$ a correct SCDD by the DOC has to be ensured for all down-stream faults. Two solution approaches are identified:

(i) optimisation of settings within device specific boundaries (e.g. for the characteristic angle),

(ii) modification of SCDD to provide robust fault type discrimination.

The second option is known from distance protection devices [10]. Fault type detection happens by comparing the three phase currents. Only those phases are considered as faulty which show a current value exceeding $2 / 3$ of the measured maximum phase current $(2 / 3$ current check).

These solutions are investigated for the first two SCDD algorithms to confirm an improvement and potential fixing of the problem (Fig. 14)

The evaluation considers the same fault cases and locations as before. For SCDD 2a, a first improvement could be reached by optimised parameter settings (2a'). For SCDD 1 and $2 \mathrm{a}-\mathrm{c}$ the modified fault type discrimination by the $2 / 3$ current check shows

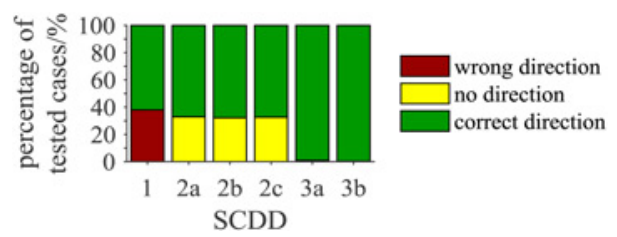

Fig. $13 S C D D$ results using the minimum permissible setting threshold $I_{>\text {forw }}$ for all 972 cases varied

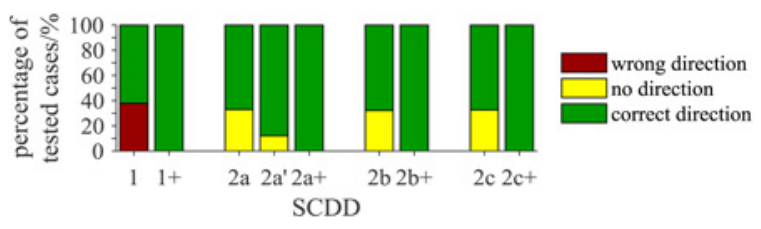

Fig. $14 S C D D$ results comparing original with improved direction evaluation criteria for all 972 variations 
a successful elimination of the problem as Fig. 14 illustrates $(1+, 2 \mathrm{a}+$ to $2 \mathrm{c}+$ ). This improvement goes without any performance reduction for other fault cases (not shown here).

Feeders that today or in the future need to utilise a DOC, should apply the maximum permissible setting for $I_{>\text {forw }}$. Those feeders should be regularly checked for potentially critical behaviour by evaluating the set $I_{>\text {forw }}$ thresholds relative to the total $I_{\text {rIIDG }}$ value of installed IIDG:

$$
I_{>\text {for w }}<I_{r \text { IIDG }}
$$

This data should be made available for protection planning personal. In general, a more detailed calculation will remedy the need to take action in most cases. This in turn necessitates adequate calculation tools and distribution system data. In cases, that need action the parameter setting of the applied DOC should be optimised to the specific grid conditions.

The suggested robust fault type discrimination offers a simple device and vendor independent approach for the development of specific firmware upgrades of commercially available DOC protection relays. Those may be selectively and thereby cost efficiently applied by DSOs. Newly installed DOC devices should be required to incorporate the suggested robust fault type discrimination or equivalent measures.

\section{Conclusion}

In case of massive integration of IIDG in LV feeders conventional fuse protection may fail to operate reliably due to blinding. Technically suitable protection solutions in dependency of the given circumstances are discussed. These are already available at the market today or are currently under development. Specifically adapted solutions with reduced complexity will most probably allow a significant cost reduction in the future.

In MV grids, digital protection relays already offer various degrees of freedom to widely avoid protection failures due to blinding by means of an individual parameterisation in the future.
In long radial feeders with high IIDG penetration unconventional usage of DOC can help sustaining reliable protection in the future. Approaches for making commercially available DOC more robust for this future application by means of parametrisation guidelines and firmware-upgrades are shown.

The necessity of performing short circuit calculations during future MV and LV protection planning becomes evident. With calculations, the full capacity of existing protection systems and IIDG integration potentials can be used and appropriate actions can be taken. Tools that allow accurate modeling of the IIDGs' current source and diverse fault behaviour need to be used. This diversity should be restricted in future by enhanced guidelines to allow efficient and reliable protection planning.

\section{Acknowledgments}

This work has partially been sponsored by the German Federal Ministry for Economic Affairs and Energy under grant 03ET7506. The authors gratefully acknowledge the various contributions by the further project partners.

\section{References}

1 Wippenbeck, T., Jäkel, M., Schmidt, T., et al.: 'Development and cross-validation of short-circuit calc. [...]'. CIRED, 2015

2 VDE: 'AR-N 4105: generators connected to the LV distribution network'. Technical Guideline, 2011

3 BDEW: 'Generating plants connected to the medium-voltage networks', Technical guideline, 2013

4 CIGRE Working Group C4.110, 2010: 'Voltage dip immunity of equipment and installations'

5 Glinka, F., Bertram, R., Wippenbeck, T., et al.: 'Protection of today's and future low voltage grids $[\ldots]$ '. DPSP, 2016

6 Bessei, H., Glinka, F., et al.: 'Smart fuses for smart grids [...]'. ICEFA, 2015

7 Koprivšek, M.: 'Triggered fuse'. ICEFA, 2015

8 German Federal Government: 'Ordinance on system services by wind energy plants', 2008

9 IEC 60909-0:2016-01: 'Short-circuit currents in three-phase a.c. systems Part 0'

10 Schneider Electric: 'Technical manual 'Easergy MiCOM P433/EN', 2016 